\title{
UNE ÉVALUATION DES PERFORMANCES ÉCONOMIQUES DE LA PÊCHE CÔTIÈRE : LE CAS DE LA BRETAGNE
}

\author{
Pascal Le Floc?h et al.
}

Armand Colin | Revue d'Économie Régionale \& Urbaine

\section{8/5 - décembre}

pages 753 à 771

\section{ISSN 0180-7307}

Article disponible en ligne à l'adresse:

http://www.cairn.info/revue-d-economie-regionale-et-urbaine-2008-5-page-753.htm

Pour citer cet article :

Le Floc?h Pascal et al., « Une évaluation des performances économiques de la pêche côtière : le cas de la Bretagne ",

Revue d'Économie Régionale \& Urbaine, 2008/5 décembre, p. 753-771. DOI : 10.3917/reru.085.0753

Distribution électronique Cairn.info pour Armand Colin.

(C) Armand Colin. Tous droits réservés pour tous pays.

La reproduction ou représentation de cet article, notamment par photocopie, n'est autorisée que dans les limites des conditions générales d'utilisation du site ou, le cas échéant, des conditions générales de la licence souscrite par votre établissement. Toute autre reproduction ou représentation, en tout ou partie, sous quelque forme et de quelque manière que ce soit, est interdite sauf accord préalable et écrit de l'éditeur, en dehors des cas prévus par la législation en vigueur en France. II est précisé que son stockage dans une base de données est également interdit. 


\section{Une évaluation des performances économiques de la pêche côtière : \\ le cas de la Bretagne*}

\section{Assessing economic performance for the coastal fishery : the case of Brittany \\ (France)}

Pascal LE FLOC'H**

UBO/UMR Amure-Cedem

2, rue de I'Université, 29334 Quimper Cedex

plefloch@univ-brest.fr

Olivier Thébaud

IFREMER/UMR Amure-Cedem

Département d'Economie Maritime

Centre de Brest - BP 70 - 29280 Plouzane

JEAN BONCOEUR

UBO/UMR Amure-Cedem

2, rue de l'Université, 29334 Quimper Cedex

Fabienne DAURÈS

IFREMER/UMR Amure-Cedem

Département d'Economie Maritime

Centre de Brest - BP 70 - 29280 Plouzane

Olivier GUYADER

IFREMER/UMR Amure-Cedem

Département d'Economie Maritime

Centre de Brest - BP 70 - 29280 Plouzane

Mots-clés : pêche côtière, performance économique, dépendance énergétique, mesures de gestion

Keywords : coastal fishery, economic performance, energy dependency,

management measures

Classification JEL : C81, Q22, R11.

*Première version mai 2007, version révisée février 2008.

Cet article est issu d'une recherche financée dans le cadre du Programme CHALOUPE:

CHAngement gLObal, dynamiqUe de la biodiversité marine exploitée et viabilité des PEche-

ries ; Projet Biodiversité ANR-IFB 2005.

Les auteurs remercient les deux rapporteurs anonymes pour leurs remarques constructives. Ils restent seuls responsables des erreurs et imperfections qui pourraient subsister.

** auteur correspondant 


\section{Résumé}

La pêche professionnelle occupe une place significative dans le tissu socioéconomique de certaines zones littorales. Les flottilles exerçant l'essentiel de leur activité dans la bande côtière méritent une attention particulière. L'activité des flottilles côtières est généralement mal connue, ce qui limite la capacité à porter un diagnostic sur leur viabilité économique. Prenant pour cas d'étude la Bretagne, cet article évalue le poids et les performances économiques de la pêche côtière. Les résultats indiquent que les performances économiques de ces flottilles ne sont pas inférieures à celles des flottilles hauturières. Les navires côtiers présentent des atouts importants, en termes d'intensité énergétique et de capacité à opérer hors financements publics. En l'absence de mesures de régulation de l'accès adaptées à leurs spécificités techniques, ils sont vulnérables à la pression exercée par les navires hauturiers sur les ressources de la bande côtière.

\section{Summary}

Fishing activities have a significant role in socio-economic base for a few coastal areas. Fleets operating mainly in coastal fisheries deserve a special interest due to their narrow links with the home base. Coastal fleet activities are not very known, restricting the capacity to deliver a diagnosis on their economic viability. This article suggests an assessment of the influence and the economic performance of the coastal fleets in Brittany. Results show similar economic performances for coastal units compared to offshore units. Furthermore, coastal units own additional advantages in relation to their energy consumption and capacity to operate without public subsidies. There are vulnerable to fishing effort of offshore fleets in the coastal zone in the absence of specific fisheries management measure.

\section{-1 -
Introduction}

Le poids économique de la pêche est généralement très faible à l'échelle européenne et nationale mais il peut être localement non négligeable. Une étude récente portant sur l'emploi dans la filière pêche, comprenant le secteur des flottilles, la transformation et l'aquaculture, évalue l'effectif employé à 421000 personnes à l'échelle de l'Union Européenne en 2002 et 2003 (SAlz et al., 2006). Si la filière des produits de la mer occupe une place modeste sur le plan national (moins de 0,3\% de l'emploi salarié en France), son insertion régionale est beaucoup plus forte en Bretagne (1 à $2 \%$ de l'emploi régional).

Cependant, la pêche n'est pas un secteur homogène, et le lien de la pêche avec le tissu socioéconomique local, ainsi que la capacité d'aménagement des pêcheries à l'échelle locale et régionale, varient fortement selon la nature de l'activité déployée en haute mer ou en bande côtière. Dans le cas de la pêche hauturière pratiquée au-delà de la bande côtière, celle-ci étant étendue à 12 milles nautiques du trait de côte (soit une extension de $22 \mathrm{~km}$ ), le lien traditionnel entre une flottille, un port de débarquement et les emplois à terre est de plus en plus volatil.

Au contraire, les unités de pêche essentiellement actives en bande côtière ont un plus fort enracinement dans l'économie locale. Ainsi, l'évaluation des performances économiques de la pêche côtière présente un intérêt particulier du point de vue du développement économique régional. En outre, la capacité de régulation publique y est également plus importante compte tenu du renforcement des mesures de 
gestion régionalisées dans la bande côtière en France, et plus particulièrement en Bretagne.

La problématique développée dans cet article repose sur la contribution économique de la pêche côtière aux revenus de la pêche artisanale bretonne, ce qui suppose de distinguer les unités de production en fonction d'un critère de localisation géographique dépendant des pêcheries exploitées (ou sites de production). Dès lors qu'il est possible de quantifier la contribution économique d'un segment de pêche selon son positionnement en mer, on peut aborder la question de la soutenabilité économique du segment considéré, notamment dans le contexte conjoncturel, voire structurel, de la forte hausse du prix de l'énergie. Il s'agit plus précisément de répondre à la question suivante : la pêche côtière est-elle en mesure, aujourd'hui, de constituer un point d'appui sérieux (bien sûr non exclusif) pour une politique régionale de gestion durable du secteur halieutique?

La première partie décrit la méthode d'identification des unités de pêche selon leur degré d'inféodation à la bande côtière. Le poids économique de la pêche côtière est évalué dans une seconde partie. La question de la soutenabilité économique des unités côtières, relativement à celle des navires hauturiers, est ensuite abordée. Enfin, la discussion s'appuie sur les arguments économiques justifiant l'idée que la pêche côtière peut servir de point d'appui à une politique régionale du secteur halieutique.

\section{-2 - \\ Mesure du degré d'inféodation à la bande côtière}

À travers le cas de la pêche bretonne, dont la part dans les débarquements de produits frais s'élève à $42 \%$ des débarquements nationaux en 2005 en volume comme en valeur (OFIMER, 2006), nous proposons une structuration de la flotte de pêche en termes de zone d'activité, plutôt qu'en termes administratifs ou physiques. Cette nouvelle définition des activités de pêche revêt un caractère innovant puisqu'elle associe des sources d'information jusque-là étanches, de nature géographique (identification de l'activité des navires par rapport à la bande côtière) et de nature économique (exploitation de données comptables).

Une matrice des degrés d'inféodation à la bande côtière est proposée par BERTHOu et al. (1999). Le critère de localisation des navires sur leurs sites de production s'appuie sur le gradient du rayon d'action des unités de pêche. Les résultats sont ainsi présentés en distinguant les navires « côtiers " (plus de $75 \%$ du temps d'activité en bande côtière), les navires " mixtes " ( $25 \%$ à $75 \%$ en bande côtière) et les navires du " large " (moins de 25 \% en bande côtière).

Ce travail s'appuie sur les bateaux de pêche localisés en Bretagne et d'une longueur inférieure à 24 mètres. Les programmes de collecte de données, dans le cadre de la Politique Commune des Pêches, distinguent globalement trois segments de flottilles en termes de longueur : les unités de moins de 12 mètres, les unités de 12 à 24 mètres, et les unités de plus de 24 mètres (Union Européenne, 2000 ; Union 
Européenne, 2001). Les deux premiers segments, navires de moins de 24 mètres, sont historiquement liés à un mode de production artisanal tel que le propriétaire du navire est également embarqué et membre de l'équipage. Si cette spécificité n'est pas systématiquement vérifiée (il existe des armements propriétaires de plusieurs unités de pêche de moins de 24 mètres), elle demeure un cas général parmi les navires de moins de 24 mètres. En revanche, les navires de plus de 24 mètres appartiennent à des armements au sein desquels le propriétaire n'est pas embarqué et dont l'insertion au sein du système productif régional est moins marquée. Ainsi, la population de la flotte de pêche bretonne de moins de 24 mètres se compose de 1406 navires actifs en 2005, représentant $92 \%$ de l'ensemble des unités de pêche immatriculées en Bretagne (BERTHOU et al., 2006).

$78 \%$ de la flottille bretonne appartiennent au segment des navires côtiers en 2005, tandis que les navires du large représentent $14 \%$ de l'ensemble des unités actives. Si la proportion de ces deux segments est stable sur la période 2000-2005, celle des unités mixtes a décru de $12 \%$ à $9 \%$ dès 2003 .

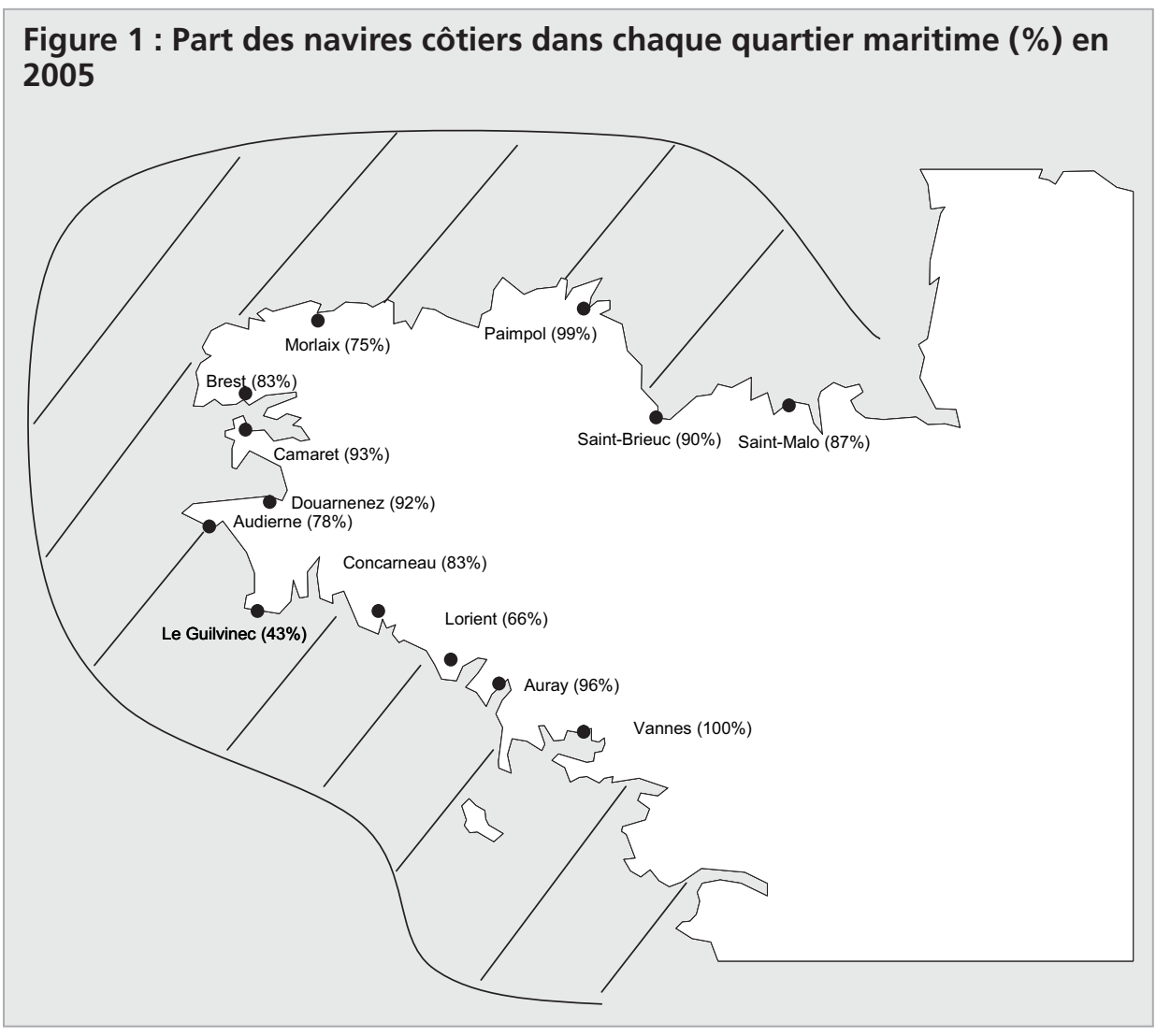

La figure 1 indique la part relative des navires côtiers dans chaque quartier maritime. À l'exception du Guilvinec, les bateaux exploitant essentiellement les ressources halieutiques en bande côtière ${ }^{1}$ représentent plus de $66 \%$ des effectifs de 
chaque quartier en 2005. Certains quartiers maritimes sont même fréquentés exclusivement par des unités côtières (Vannes et Paimpol, accueillant une centaine de navires).

La répartition des navires de moins de 24 mètres indique une concentration importante des unités, tous segments de localisation confondus, dans le quartier maritime du Guilvinec, soit un cinquième de la population des navires immatriculés en Bretagne. Etendu sur quatre sites portuaires (Loctudy, Lesconil, Le Guilvinec, Saint-Guénolé), ce quartier maritime tire sa spécificité principalement de la production débarquée par les navires du large. En revanche, la présence des unités côtières à l'échelle de la région est beaucoup plus homogène. Par exemple, les navires côtiers sont situés à parts équivalentes entre la Bretagne nord (48\% de l'effectif), associant les quartiers de Camaret à Saint-Malo, et la Bretagne sud (52\% de l'effectif), délimitée par les quartiers de Douarnenez et de Vannes. Enfin, les trois principaux quartiers maritimes (Saint-Brieuc, Auray et Le Guilvinec) concentrent $37 \%$ de l'effectif des unités côtières.

Cette répartition spatiale de la composante côtière de la flottille bretonne n'est pas sans intérêt en termes d'aménagement du littoral (LATOUCHE et LE FLOC'H, 2000). En effet, la distribution homogène de la population des navires côtiers traduit, d'une part, la polyvalence de cette activité, et, d'autre part, le maintien d'une part essentielle des emplois à la pêche sur l'ensemble du littoral breton. L'effectif moyen de l'équipage en 2005 à bord des navires côtiers est de 2,2 hommes, tandis qu'il est de 4,1 sur les navires mixtes et de 5,4 sur les bateaux du large. On peut dès lors estimer que la part de l'emploi à la pêche sur les unités côtières s'élève à $63 \%$ de l'emploi total de la flottille artisanale bretonne de moins de 24 mètres en 2005 . Il faut également souligner le degré élevé de polyvalence des navires côtiers, à l'opposé de la forte spécialisation des navires mixtes et du large (pratiquant majoritairement l'activité du chalutage, engin de pêche trainé par l'arrière du navire, et à un degré moindre l'activité du filet).

Si la présence des navires côtiers sur l'ensemble du littoral présente des atouts en termes d'aménagement et d'emploi, il reste néanmoins à vérifier le poids économique de cette composante de la flottille par le biais de la structure des coûts d'exploitation et des revenus générés.

\section{- 3 - \\ Le poids économique de la pêche côtière}

L'ensemble des unités de moins de 24 mètres présentes dans la base de données comptable de l'Observatoire économique régional des pêches de Bretagne 2 en 2005 a été pris en compte, soit un total de 463 navires (BIHEL et LE FLOC'H, 2006). Les navires identifiés selon le gradient du rayon d'action représentent le tiers de l'effectif de la flotte de pêche artisanale. Le taux d'échantillonnage est de $27 \%$ pour les navires " côtiers », atteint $77 \%$ pour les unités « mixtes », et $38 \%$ pour les bateaux du « large». 
Les navires localisés majoritairement en bande côtière sont plus âgés (22,7 ans) que les autres unités (tableau 1). Les bateaux "côtiers" sont essentiellement des unités de moins de 12 mètres et leurs capacités de production moyennes sont six fois plus réduites que celles des navires du large en termes de tonnage, et trois fois plus faibles en termes de puissance motrice.

\begin{tabular}{|c|c|c|c|c|c|c|c|}
\hline Gradient & $\begin{array}{l}\text { Nombre } \\
\text { de navires } \\
\text { dans la } \\
\text { base }\end{array}$ & & $\begin{array}{l}\text { Âge en } \\
2005 \\
\text { (années) }\end{array}$ & $\begin{array}{l}\text { Longueur } \\
\text { (m) }\end{array}$ & $\begin{array}{l}\text { Tonnage } \\
\text { (tjb) }\end{array}$ & $\begin{array}{l}\text { Puissance } \\
(\mathrm{kw})\end{array}$ & $\begin{array}{l}\text { Equipage } \\
\text { (nombre de } \\
\text { personnes) }\end{array}$ \\
\hline $\begin{array}{l}\text { Navires } \\
\text { côtiers }\end{array}$ & 292 & $\begin{array}{l}\text { - moyenne } \\
\text { - écart- } \\
\text { type }\end{array}$ & $\begin{array}{l}22,7 \\
8,9\end{array}$ & $\begin{array}{l}10,2 \\
2,3\end{array}$ & $\begin{array}{l}11,6 \\
8,9\end{array}$ & $\begin{array}{l}126,1 \\
60,9\end{array}$ & $\begin{array}{l}2,2 \\
1,2\end{array}$ \\
\hline $\begin{array}{l}\text { Navires } \\
\text { mixtes }\end{array}$ & 70 & $\begin{array}{l}\text { - moyenne } \\
\text { - écart- } \\
\text { type }\end{array}$ & $\begin{array}{l}19,1 \\
8,2\end{array}$ & $\begin{array}{l}14,6 \\
2,7\end{array}$ & $\begin{array}{l}31,7 \\
16,0\end{array}$ & $\begin{array}{l}236,3 \\
81,9\end{array}$ & $\begin{array}{l}4,1 \\
1,3\end{array}$ \\
\hline $\begin{array}{l}\text { Navires du } \\
\text { large }\end{array}$ & 101 & $\begin{array}{l}\text { - moyenne } \\
\text { - écart- } \\
\text { type }\end{array}$ & $\begin{array}{l}17,5 \\
5,4\end{array}$ & $\begin{array}{l}20,2 \\
3,3\end{array}$ & $\begin{array}{l}72,4 \\
29,4\end{array}$ & $\begin{array}{l}372,2 \\
102,7\end{array}$ & $\begin{array}{l}5,4 \\
1,4\end{array}$ \\
\hline
\end{tabular}

Si on croise la typologie élaborée par BERTHOU et al. (1999) pour caractériser le gradient du rayon d'action des navires avec le critère de la longueur des navires de l'échantillon, on constate que l'activité en bande côtière relève principalement de navires disposant d'une longueur inférieure à 12 mètres (tableau 2). Dans le cas opposé, l'activité de la pêche majoritairement hors bande côtière, concerne surtout les unités de plus de 16 mètres (75\% des unités de 16 à 24 mètres).

\section{Tableau 2 : Répartition des navires de l'échantillon selon la longueur et le rayon d'action}

\begin{tabular}{l|r|r|r}
$\begin{array}{l}\text { Classe de longueur } \\
\text { Rayon d'action }\end{array}$ & $<\mathbf{1 2} \mathbf{~ m}$ & [12 $\mathbf{~ m - 1 6 ~} \mathbf{~}$ [ & [16 $\mathbf{m}-\mathbf{2 4}$ m.[ \\
\hline Côtiers & $93 \%$ & $49 \%$ & $6 \%$ \\
\hline Mixtes & $6 \%$ & $34 \%$ & $18 \%$ \\
\hline Large & $0 \%$ & $17 \%$ & $75 \%$ \\
\hline Total & $100 \%$ & $100 \%$ & $100 \%$
\end{tabular}

La situation économique des armements artisans bretons, en 2005, est décrite à travers la ventilation du chiffre d'affaires (figure 2). Le montant moyen du chiffre d'affaires varie de $150 \mathrm{k} €$ pour les unités côtières à $640 \mathrm{k} €$ pour les unités du large. Celles-ci réalisent donc une production en valeur 4,2 fois plus élevée que celle des bateaux présents majoritairement en bande côtière. La part des consommations 
intermédiaires et des taxes dans le montant du chiffre d'affaires fluctue de $37 \%$ pour les navires côtiers à $51 \%$ pour les navires du large. Si la rémunération brute de l'équipage se situe autour de $40 \%$ du chiffre d'affaires pour les trois segments, elle reste généralement en deçà de $30 \%$ chez les navires côtiers de taille inférieure à 9 mètres. Le mode de rémunération de l'équipage, traditionnellement fondé sur le système à la part, n'est pas toujours appliqué sur les unités de moins de 12 mètres. Sur les navires ayant un seul homme à bord, l'excédent brut d'exploitation (EBE) englobe généralement le revenu du travail. Enfin, la part du chiffre d'affaires consacré à la rémunération de l'armement (Excédent brut d'exploitation et primes d'armement) décroît avec le rayon d'action des navires ( $12 \%$ chez les unités du large contre $23 \%$ chez les côtiers).

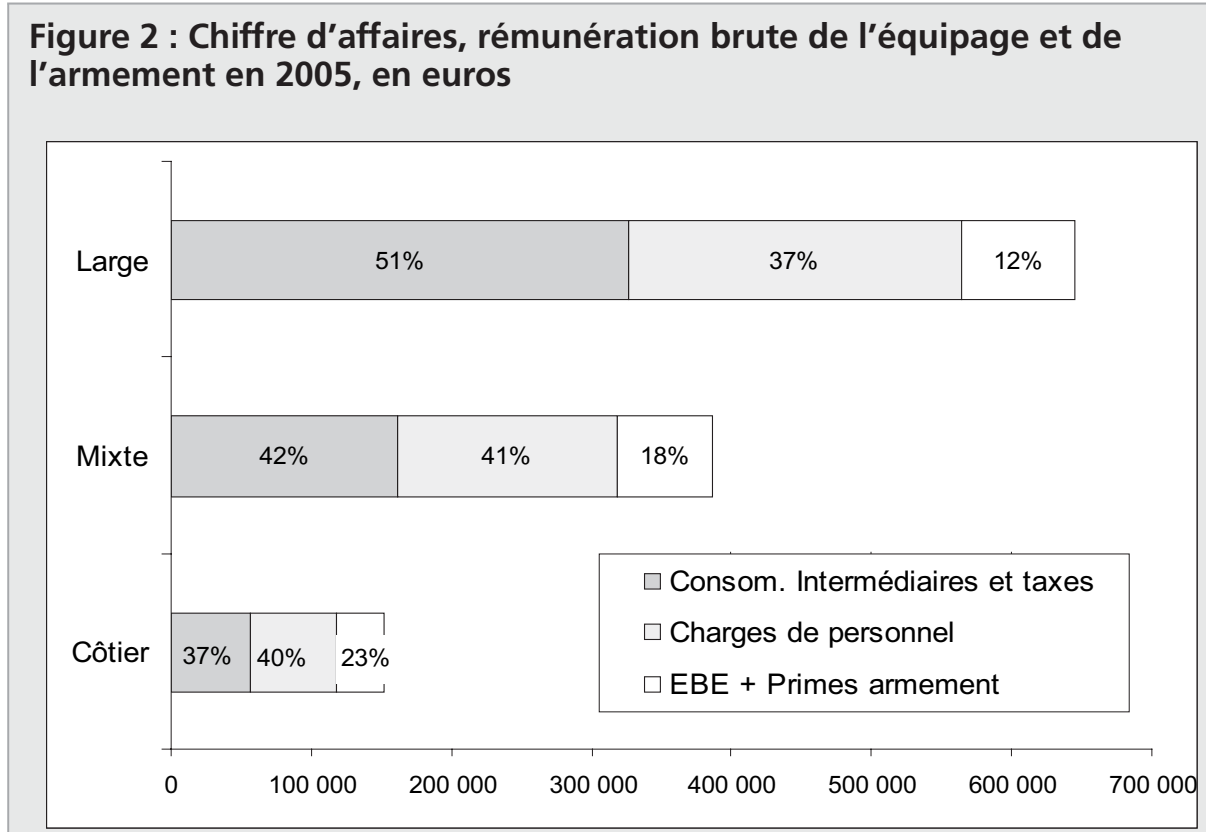

Dans l'optique de mesurer la contribution de chaque segment à la création de richesse, la comparaison des performances économiques des unités de pêche s'appuie sur la valeur ajoutée brute (VAB) unitaire ${ }^{3}$. Considérée comme un indicateur du niveau de production, au même titre que le chiffre d'affaires, la valeur ajoutée brute est rapportée à deux indicateurs d'utilisation des facteurs de production (figure 3). Le facteur travail est appréhendé selon le nombre d'hommes embarqués. Le facteur capital est approché à partir de la jauge. Ainsi, le rapport de la valeur ajoutée brute à un facteur de production physique fournit une mesure de la productivité apparente. À la différence de la productivité appréhendée à partir de la production brute (ou chiffres d'affaires), la mesure de performance obtenue en fonction de la valeur ajoutée brute exclut les facteurs intermédiaires (détruits au cours du cycle de production). Les consommations intermédiaires incluent notamment les dépenses en carburant-lubrifiant, les vivres, la glace, le matériel de pêche, 
les frais d'entretien-réparations, l'assurance. Ces facteurs associent donc des biens et des services.

Les performances économiques de la pêche côtière soutiennent la comparaison avec celles de l'activité hauturière (navires mixtes et du large) car, si la productivité apparente du travail y est plus faible, la productivité apparente du capital y est plus forte.

\section{Figure 3 : Productivité apparente du travail (par homme embarqué) et du} capital (tjb) en euro, année 2005

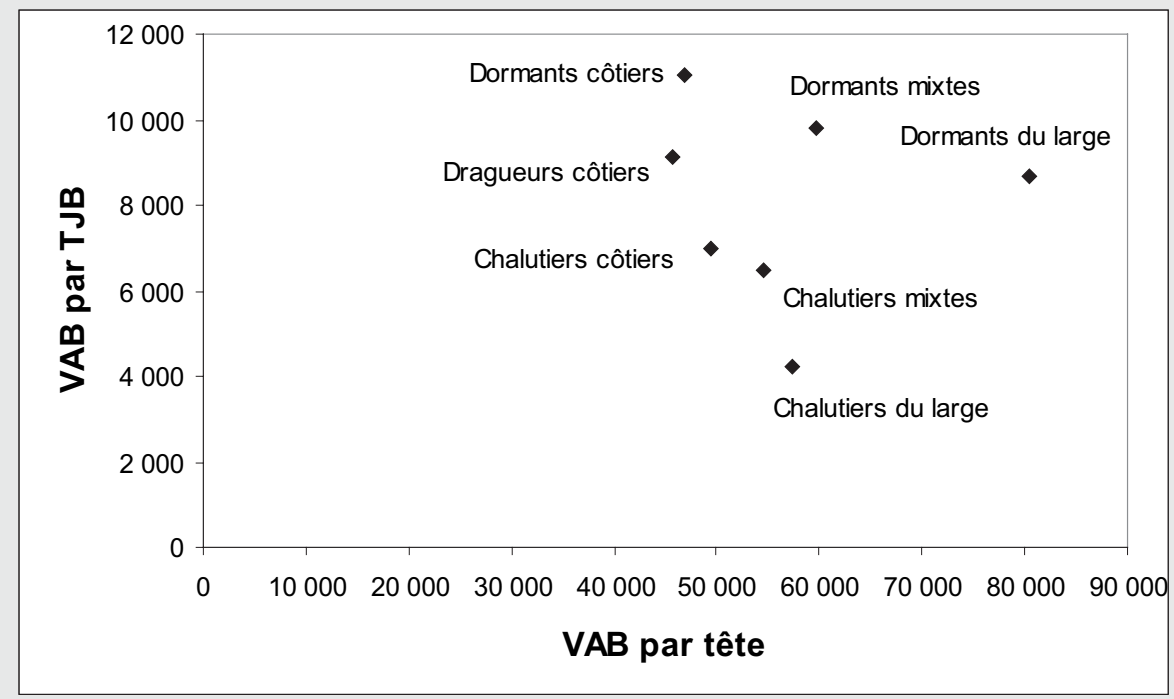

Des travaux antérieurs sur la mesure des indicateurs de performance économique ont, par ailleurs, démontré la pertinence de rapporter ces indicateurs à une intensité de pêche, tel que le temps passé en mer appréhendé soit par le nombre annuel de jours de mer ou le nombre annuel des heures du moteur (BONCOEUR et al., $2000 a$ ). Dans ce cas, les performances des unités côtières sont renforcées compte tenu d'une durée d'utilisation plus faible des moyens de production. En effet, les données d'enquête économiques collectées dans le cadre du SIH (Système d'Information Halieutique) indiquent que la durée annuelle moyenne des navires de plus de 12 mètres pratiquant le chalutage est proche de 240 jours (IFREMER, 2006). Cette durée ne dépasse pas 215 jours pour les unités de moins de 12 mètres, et est même inférieure à 200 jours chez les navires côtiers utilisant les engins dormants (filet, ligne, casier).

Finalement, la contribution des navires côtiers à la formation de la VAB à l'échelle régionale des navires de moins de 24 mètres peut être estimée sur la base des performances économiques de l'échantillon pour l'année 2005. Les résultats économiques moyens par navire, calculés sur la base de la valeur ajoutée brute, vont 
de $103 \mathrm{k} €$ chez les unités de la pêche côtière à $319 \mathrm{k} €$ chez les bateaux du large. Les unités mixtes ont produit une richesse brute de $231 \mathrm{k} €$. Ces résultats sont extrapolés à la population des navires actifs en Bretagne pour l'année 2005 sur la base de l'échantillon. Estimée à 201 millions d'euros, la VAB produite par les navires de moins de 24 mètres en 2005 représente $0,3 \%$ de la $\mathrm{VAB}$ totale de la région Bretagne $^{4}$ (INSEE, 2007) et $7 \%$ de la VAB de la branche " Agriculture, sylviculture, pêche ». La figure 4 indique la répartition de la VAB totale des navires bretons de moins de 24 mètres en fonction du rayon d'action. La pêche professionnelle en bande côtière contribue plus fortement à la richesse brute des unités artisanales de moins de 24 mètres que les navires mixtes et du large. Sa part s'élève à $56 \%$ de la VAB totale en 2005.

\section{Figure 4 : Répartition de la VAB totale des navires bretons de moins de 24 mètres en 2005}

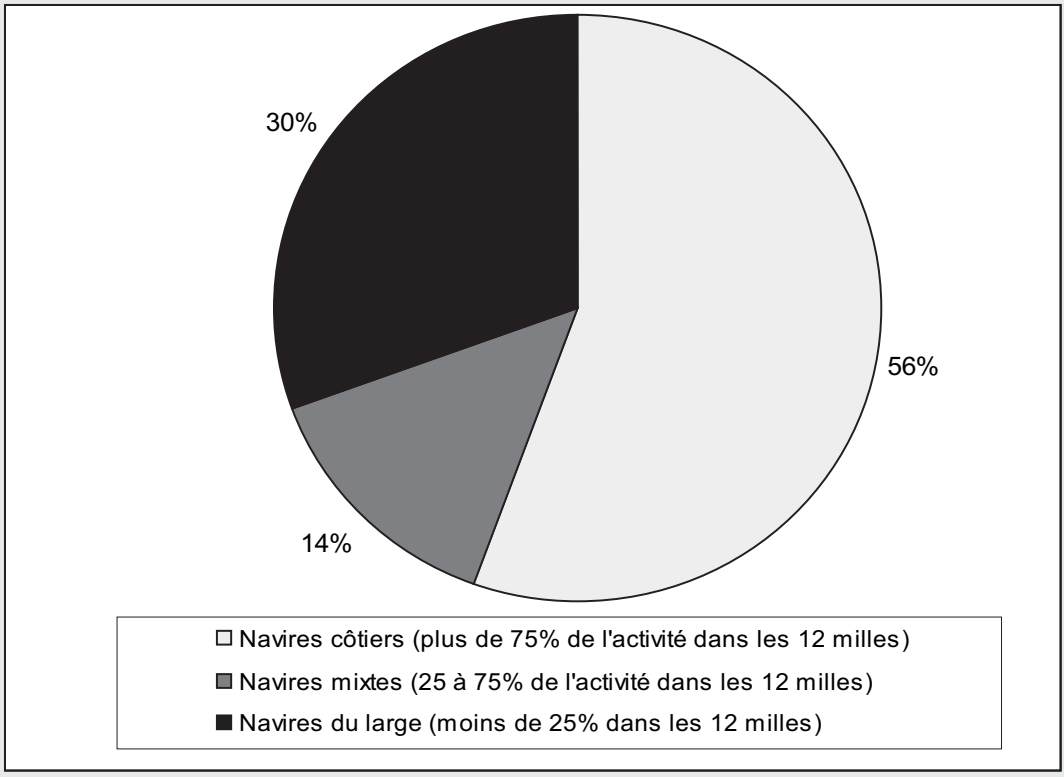

Source : Observatoire économique régional des pêches \& Ifremer

La contribution importante de la pêche côtière à la création de richesse de l'ensemble des navires bretons s'explique également par un impact moindre des dépenses en carburant comparativement aux navires exploitant des pêcheries situées au-delà de la bande côtière. Il est, par conséquent, pertinent d'étudier la dépendance énergétique des navires de pêche selon leur rayon d'action (LE FLOC'H et al., 2006). Cette dépendance souligne, en effet, une plus grande soutenabilité économique de l'activité des unités côtières par rapport aux unités hauturières. 


\section{-4 - \\ La soutenabilité économique de la pêche côtière}

On considère comme soutenable économiquement l'activité de pêche la moins dépendante de la consommation de carburant. Si le poids du composant énergétique représente structurellement une part significative dans la structure des coûts d'exploitation des navires de pêche, la tendance à la hausse du coût du carburant depuis 2000 a entraîné une plus forte détérioration des performances économiques des navires hauturiers. Ces derniers sont également devenus plus dépendants des mesures conjoncturelles de soutiens publics.

\section{Figure 5. Ventilation du chiffre d'affaires moyen en 2005}

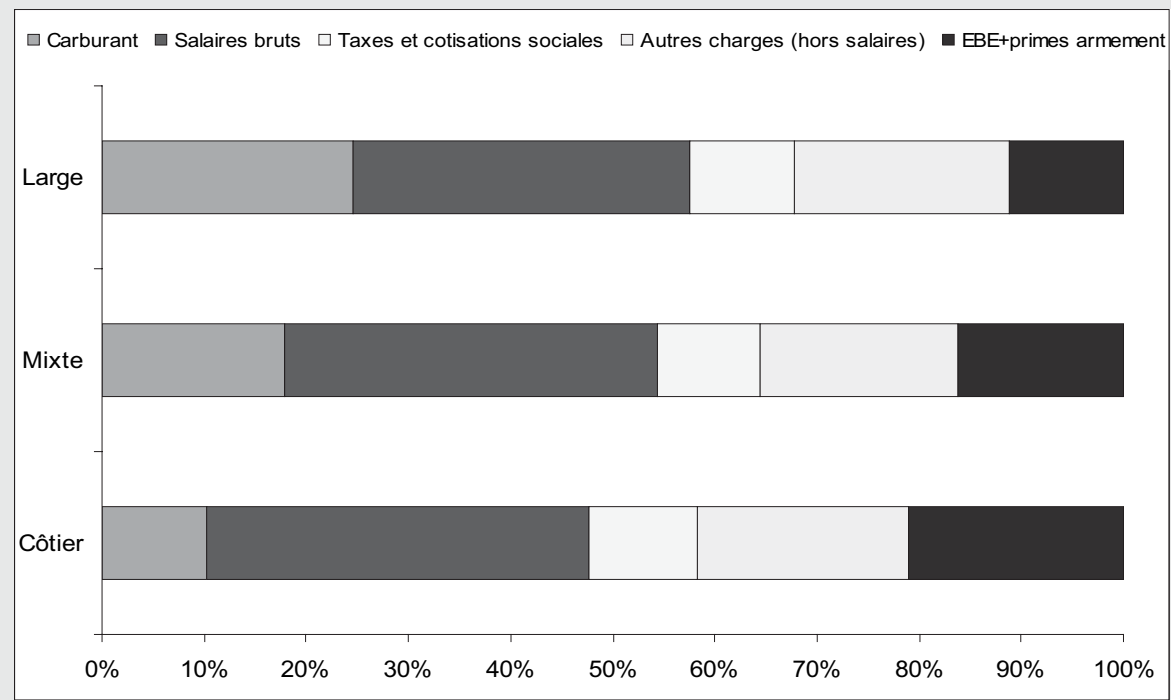

Source : Observatoire économique régional des pêches

Ainsi, les navires du large sont les plus affectés par la forte hausse du prix du carburant, constatée en 2005. En effet, la ventilation du chiffre d'affaires (CA) moyen met en avant le poids du composant énergétique chez les navires du large, et à l'inverse la part plus faible de la rémunération de l'armement (EBE et primes armement) chez ces mêmes armements (figure 5). À l'opposé, les côtiers sont faiblement sensibles à la dépense de carburant mais consacrent plus de $20 \%$ du chiffre d'affaires à la rémunération de l'armement. La situation en 2005 a été profondément modifiée en raison de la hausse importante du prix du carburant pour les flottilles consacrant moins de $25 \%$ de leur temps de pêche en bande côtière. Ainsi, la part des dépenses énergétiques s'est élevée à $25 \%$ du chiffre d'affaires chez les unités du large, soit sept points de plus qu'en 2004. Si cette augmentation est moins marquée chez les unités mixtes, elle reste cependant significative (18\% du CA en 2005). Les rémunérations de l'équipage et de l'armement n'ont pas été affectées. Les autres charges d'exploitation (hors salaires et 
carburant) ont compensé, par une baisse d'ampleur similaire à la hausse des dépenses de carburant, cette élévation du poste énergétique dans la ventilation du chiffre d'affaires.

L'évolution négative de ces autres charges en 2005, par rapport à 2004, trouve son explication dans la mise en place d'un mécanisme de compensation de la hausse du coût du carburant ${ }^{5}$. Un outil de prévention des aléas à la pêche (Fonds de Prévention des Aléas Pêche, FPAP) a été institué en novembre 2004. Avec pour vocation principale de réduire l'impact du prix du carburant, ce mécanisme a fonctionné sur le principe d'une aide financière de l'État, dénoncée comme une atteinte au droit de la concurrence par la Commission Européenne ${ }^{6}$.

La figure 6 décrit l'impact de cette mesure de compensation sur le coût (brut et net) du facteur énergétique en 2004 et 2005. La part du coût net du carburant est identique en 2005 par rapport à 2004 pour les navires du large (18\%) et les navires côtiers ( $8 \%$ ). Cette part a même reculée d'un point chez les navires mixtes (13\% en 2005 au lieu de $14 \%$ en 2004).

\section{Figure 6 : Évolution 2004-2005 du coût brut et net du carburant en \% du chiffre d'affaires}

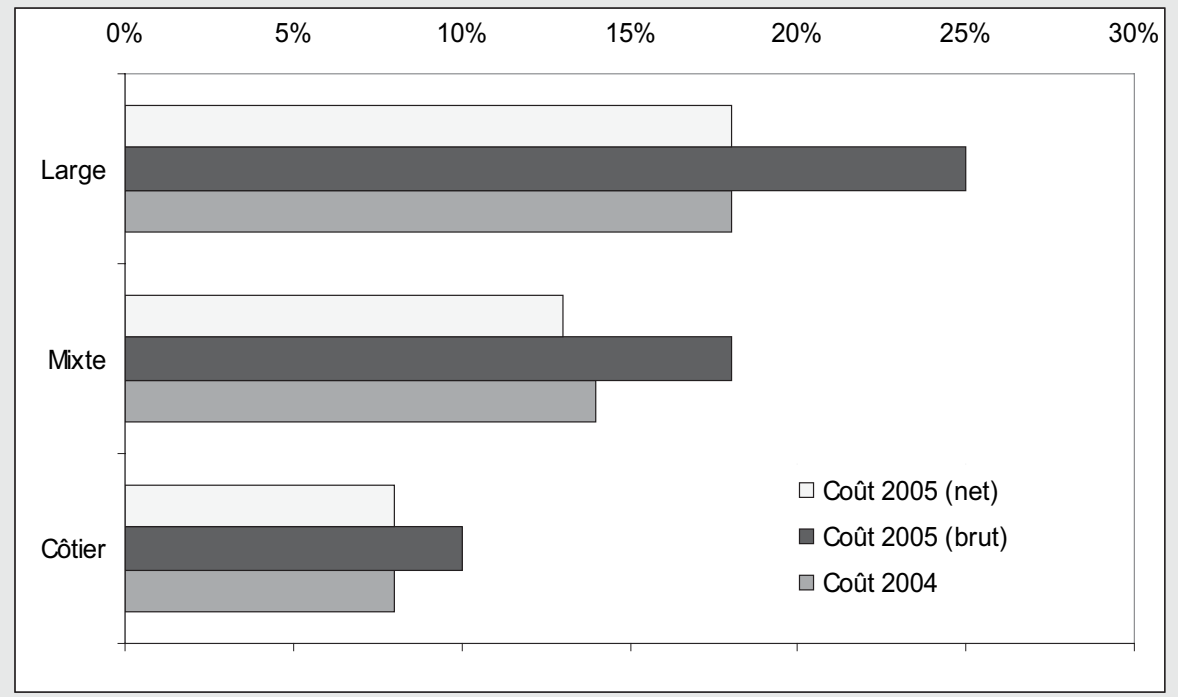

Source : Observatoire économique régional des pêches

Si les tensions inflationnistes liées au prix du pétrole sont généralement ressenties comme un phénomène conjoncturel, le suivi rétrospectif des revenus à la pêche sur la période 1998-2005 fait apparaître des tendances d'évolution différentes selon le critère du rayon d'action « côtier » et « mixte » d'un côté, et « large » de l'autre. 
Pour mieux appréhender les caractéristiques économiques de l'activité halieutique hors et en bande côtière, il paraît utile de suivre les tendances d'évolution de la rémunération des facteurs de production travail et capital.

La valeur des débarquements (ou chiffre d'affaires) a progressé de 2000 à 2003 pour les trois segments. Depuis 2003, elle progresse faiblement chez les mixtes et fléchit légèrement chez les côtiers. En revanche, les unités du large enregistrent une baisse significative de leur chiffre d'affaires, atteignant en termes réels un niveau inférieur en 2005 à celui réalisé en 1998 (-5,5\% de réduction entre ces deux périodes). La rémunération des équipages, basée sur le système du salaire à la part, révèle une évolution proche de celle de la production (figure 7a). Cependant, l'ampleur des variations des revenus du travail est amplifiée par rapport à celle du chiffre d'affaires. Sur la période 1998-2000, la baisse des salaires de 6,5 \% accompagne une diminution de la production de $2 \%$ chez les armements côtiers. Les équipages embarqués sur les navires du large accusent un recul de $15 \%$ de leurs rémunérations alors que le chiffre d'affaires est réduit de 6,6\%. Seules les unités mixtes maintiennent leur niveau de production avec, toutefois, une détérioration de $4 \%$ des salaires bruts.

\section{Figure 7. Indice de la rémunération annuelle brute moyenne de l'équipage (a) et de l'armement (b) : évolution 1998-2005, en euros 2005}
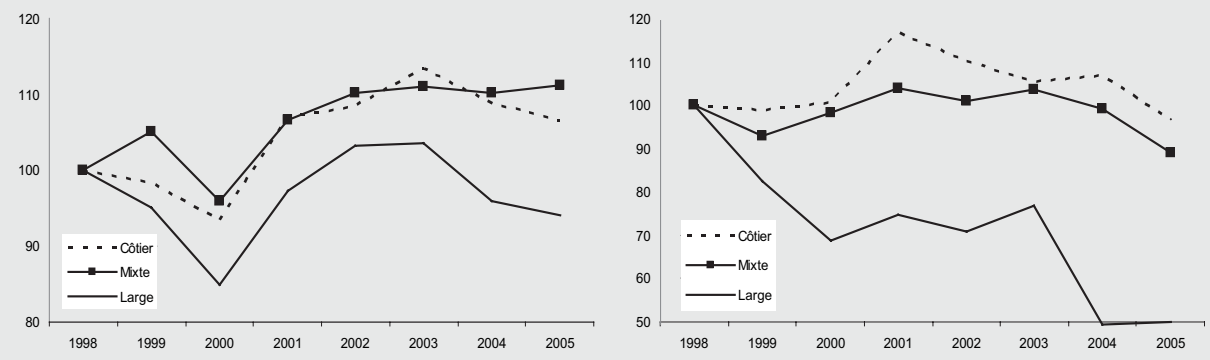

Source : Observatoire économique régional des pêches

La tendance à la baisse des rémunérations du travail est liée en partie à l'élévation du coût du carburant. En effet, le calcul des salaires des marins s'effectue sur la base du chiffre d'affaires déduit de charges communes à l'équipage, dont les dépenses de combustible. La hausse du prix du gazole en 2000 explique donc cette première phase d'évolution. De 2000 à 2003, les rémunérations s'améliorent et dépassent les niveaux de 1998. Cette phase de croissance commune aux trois segments laisse place à des évolutions contrastées de 2003 à 2005. Les équipages des unités mixtes conservent un niveau de revenu identique alors que la tendance s'inverse pour les côtiers et les navires du large. Si la situation ne soulève pas d'inquiétudes particulières chez les côtiers, elle apparaît plus critique chez les bateaux du large. Les salaires versés aux équipages de ces unités en 2005 sont inférieurs de 6 \% par rapport à la situation en début de période. Au contraire, la rémunération a progressé de $7 \%$ en 2005 par rapport à l'année 1998 pour les équipages des côtiers et de 11 \% pour ceux des navires mixtes. 
On observe une nette dégradation de la rémunération de l'armement (EBE et primes armement) sur l'ensemble de la période chez les bateaux du large (figure 7b). Les dépenses énergétiques ont été multipliées par 2,5 en 2005 par rapport à 1998 chez les navires hauturiers. Ce phénomène se traduit par une baisse de la rémunération de l'armement laquelle atteint en moyenne annuelle (taux de croissance instantané) 8,5\%. Cette évolution s'accélère en fin de période avec la baisse du chiffre d'affaires.

Afin de mieux cerner l'impact du carburant sur les revenus à la pêche, on propose d'analyser le comportement des bateaux selon leur rayon d'action à partir d'un modèle économétrique, sur la base des trois échantillons constants présentés précédemment dans le tableau 1. Un degré de liberté d'une année a été conservé dans la constitution des échantillons de telle manière qu'un navire appartient à l'échantillon constant s'il est renseigné sur la période au moins sept années sur huit. On dispose de séries continues de données comptables. L'effectif de navires côtiers fluctue entre 93 et 99 unités sur la période d'étude, celui des navires mixtes est compris entre 27 et 30 unités, enfin l'effectif des navires du large varie de 53 à 59 unités.

La consommation physique du carburant, exprimée en log (log $\left.C a r b_{t}\right)$, est expliquée sur la base de trois variables continues, la VAB $\left(\log v a b_{t}\right)$, l'âge du navire $\left(\log \hat{a} g e_{t}\right)$ et la capacité de jauge $\left(\log t j b_{t}\right)$. Deux variables binaires, identifiant les navires côtiers et les navires mixtes, sont intégrées au modèle. La variable représentant les navires du large est considérée comme la variable de référence, et est, par conséquent, retirée du modèle afin d'éviter le problème de multi-colinéarité entre variables qualitatives.

(1) $\log \left(\mathrm{Carb}_{t}\right)=\alpha+\beta \log \left(v a b_{t}\right)+\gamma \log \left(t j b_{t}\right)+\delta \log \left(\hat{a g e} e_{t}\right)+\lambda_{1}$ Côtiers $+\lambda_{2}$ Mixtes $+\varepsilon$

Les paramètres $\beta$, $\gamma$ et $\delta$ sont des coefficients d'élasticités mesurant respectivement l'impact de la variation en pourcentage de la vab, de la jauge (tjb), et de l'âge des navires sur la consommation physique de carburant (en litres). L'interprétation des coefficients des variables binaires $\left(\lambda_{1}, \lambda_{2}\right)$ repose sur la significativité et le sens par rapport à la variable de référence (les unités du large). Les résultats sont présentés dans le tableau 3.

\begin{tabular}{|c|c|c|c|c|c|c|c|c|}
\hline & 1998 & 1999 & 2000 & 2001 & 2002 & 2003 & 2004 & 2005 \\
\hline $\mathbf{R}^{2}$ & 0.91 & 0.91 & 0.90 & 0.90 & 0.90 & 0.89 & 0.88 & 0.82 \\
\hline Constante & $-2.29 *$ & -1.28 & -0.64 & -0.38 & 0.02 & -0.83 & $-2.61^{*}$ & -0.23 \\
\hline $\log \left(v a b_{t}\right)$ & $0.68 * * *$ & $0.61 * * *$ & $0.60 * \star *$ & $0.58 * * *$ & $0.57 * * *$ & $0.66 * * *$ & $0.83 * * \star$ & $0.75 * * \star$ \\
\hline $\log \left(a \hat{g e} e_{t}\right)$ & $0.34 * * *$ & $0.29 * * *$ & $0.29 * *$ & 0.27 * & 0.13 & 0.15 & 0.27 ** & 0.06 \\
\hline $\log \left(t j b_{t}\right)$ & $0.80 * * *$ & $0.85^{* * *}$ & $0.89 * * *$ & $0.87^{\star * \star}$ & $0.86^{* * *}$ & $0.78^{* * \star}$ & $0.63^{* * *}$ & $0.67 * * *$ \\
\hline Côtier & -0.17 & $-0.25 * *$ & $-0.38 * * *$ & $-0.34 * * *$ & $-0.38 * * *$ & $-0.41 * * *$ & $-0.55^{* \star *}$ & $-0.74 * \star \star$ \\
\hline Mixte & -0.03 & -0.06 & -0.15 & -0.05 & -0.11 & -0.10 & -0.14 & -0.25 \\
\hline
\end{tabular}


Les variables continues sont significatives sur l'ensemble de la période, à l'exception de l'âge des navires en 2002, 2003 et 2005. On remarque une légère dégradation du modèle en 2004 et plus particulièrement en 2002, le R² décroît de 90 \% à $82 \%$.

L'effet attendu d'un impact positif de ces variables sur la consommation physique de carburant est vérifié. Ainsi, une amélioration de $10 \%$ de la valeur ajoutée brute $\left(v a b_{t}\right)$ se traduit par une utilisation énergétique accrue de 6 à $7 \%$ de 1998 à 2003. L'effet est porté à $8 \%$ sur les deux dernières années. Le critère technique, identifié par la capacité de jauge $\left(t j b_{t}\right)$, montre qu'une élévation de cette capacité de l'ordre de 10 \% entraîne, en toute logique, un besoin supplémentaire de carburant de 8 à $9 \%$ de 1998 à 2003. Contrairement à la VAB, l'impact est réduit en 2004 et 2005, atteignant 6 à $7 \%$. Le vieillissement des unités de pêche peut également être interprété par un comportement plus " énergivore ». Toutefois, la variable âge n'est significative au seuil de $1 \%$ qu'en 1998 et 1999. En effet, la durée de vie moyenne d'un moteur ne dépasse guère 10 ans dans le cas des flottilles pratiquant les arts traînants (chalut, drague), celle-ci étant plus variable pour les navires utilisant les arts dormants (filet, casier, ligne). Par conséquent, la durée d'existence d'une unité de pêche, qui est ici prise en compte et non pas celle du moteur (dont la donnée est indisponible), n'est qu'un indicateur imparfait de la relation entre l'âge de l'outil de production et le besoin énergétique.

\section{Figure 8. Estimateurs de la variable binaire "Côtier " et prix du carburant en euro constant 2005 par litre : évolution 1998-2005}

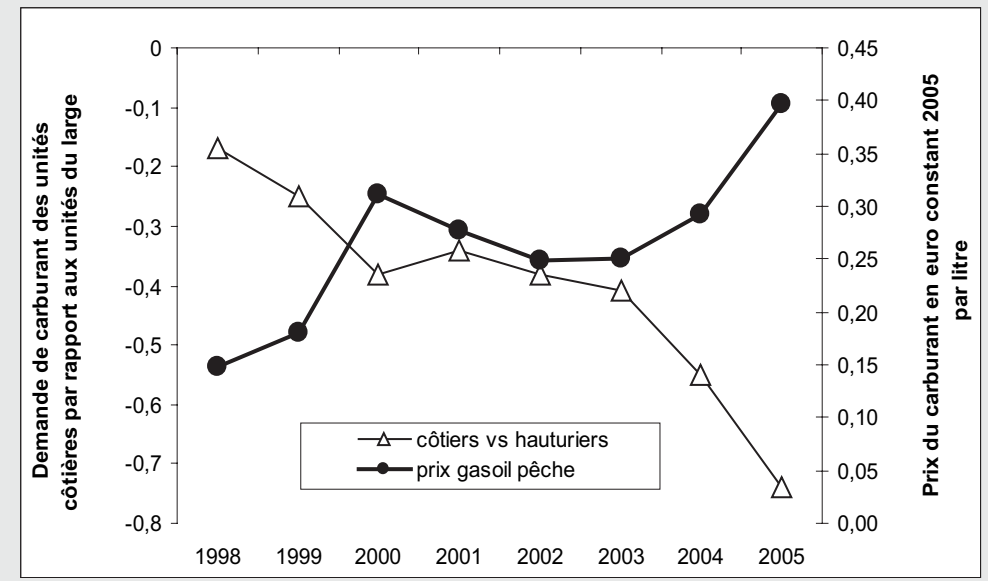

Source : Observatoire économique régional des pêches

Si les variables binaires ne peuvent directement donner lieu à des enseignements quantitatifs quant à leur impact sur la consommation de carburant, elles fournissent, en revanche, des indications claires sur la nature comportementale de chaque segment vis-à-vis du segment de référence (ici les unités du large ou unités hauturières). Mis à part l'année 1998, l'estimateur représentant les navires côtiers devient 
significatif au seuil de $5 \%$ en 1999 et au seuil de 1 \% les années suivantes. Le signe négatif révèle une plus faible utilisation de carburant par les bateaux côtiers à l'égard de la flottille du large. Les unités mixtes présentent également un signe négatif mais non significatif, ce qui ne permet pas de conclure sur une différence de comportement entre unités mixtes et unités du large. Autre élément d'importance concernant les navires côtiers, leur besoin énergétique comparé à celui des navires hauturiers diminue graduellement sur toute la période et plus fortement dès 2003, alors que le prix du gasoil à la pêche passe de 0,25 €/litre à 0,4 €/litre en 2005.

La figure 8 indique une évolution symétrique mais de sens opposé entre, d'une part, le comportement de la flottille côtière à l'égard de celle du large, représenté par les estimateurs de la variable binaire "Côtier" du modèle économétrique, et, d'autre part, le prix du gasoil à la pêche.

L'augmentation du prix de l'énergie s'inscrit de plus en plus dans un contexte structurel (BROOK et al., 2004 ; MAURICE, 2001) que conjoncturel, renforçant l'attractivité des techniques de pêche les moins consommatrices en carburant. C'est dans cette perspective que se dessine une meilleure soutenabilité économique de la pêche côtière, notamment dans une moindre dépendance à l'égard de soutiens extérieurs, en particulier d'aides publiques compensant partiellement ou totalement la hausse du coût énergétique, sans justification économique de ces aides (Union Européenne, 2006).

\section{- 5 - Conclusion}

Si l'activité de la pêche s'inscrit clairement dans le développement économique régional (ADAM, 1968 ; CHARLES, 1992) ${ }^{7}$, la contribution des pêcheries côtières à la production de l'ensemble des flottilles d'une région est rarement évaluée. Cet article montre, en effet, que la pêche côtière bretonne n'est ni anecdotique, ni économiquement dépassée (par rapport à la pêche hauturière). Compte tenu de son intérêt particulier pour le développement régional, ce secteur mérite donc l'attention des décideurs publics à l'échelle de la région.

Dans l'évaluation économique de la pêche côtière, deux points essentiels ont été soulignés. Tout d'abord, les performances économiques des navires côtiers soutiennent la comparaison avec celles des navires hauturiers (BONCOEUR et al., 2000b). Les productivités apparentes sont tout à fait comparables, voire supérieures concernant le capital physique. La pondération de ces indicateurs de performance avec la durée d'utilisation des moyens de production ne ferait qu'accentuer cette comparaison au profit des navires côtiers. En effet, en appréhendant le temps de pêche en termes de jours de mer ou à partir de la durée d'utilisation du moteur (heures moteur) par navire, la performance des unités côtières se trouve renforcée compte tenu d'une durée d'activité plus courte que celle des navires hauturiers. Cette image reste cependant imparfaite, car le temps passé en mer ne se confond pas avec le temps de pêche. Il inclut, en effet, le temps consacré à faire route vers les lieux de pêche et à en revenir. Qu'il soit mesuré en jours ou en heures, le temps annuel 
moyen de navigation augmente généralement avec la taille des navires. La prise en compte de la durée d'utilisation des facteurs de production (productivités journalières ou horaires) modifie ainsi l'image donnée par les productivités simples, surtout si l'on raisonne en termes d'heures de mer. Elle atténue, voire inverse la tendance à l'accroissement de la productivité du travail avec l'échelle de production. La productivité horaire du travail a tendance à varier en sens inverse de la taille du navire.

Le second point concerne la dépendance énergétique des flottilles selon leur rayon d'action. Dans un contexte de hausse du prix de l'énergie, la moindre dépendance des flottilles côtières à l'égard du carburant rend plus soutenable économiquement l'activité d'exploitation des ressources halieutiques en bande côtière. Les mesures conjoncturelles de soutiens publics mises en place en 2000, puis en 2004 et plus récemment en 2008, prennent la forme d'allègements de charges sociales, d'exonération de redevances portuaires ou d'aides directes, comme l'application d'une taxe sur le prix du poisson au débarquement (celle-ci étant alors supportée par le consommateur). Le mécontentement exprimé par les pêcheurs à chaque hausse brutale du prix du carburant relève essentiellement du cas des navires hauturiers pratiquant la technique du chalutage. Les réponses apportées par les décideurs politiques s'appuient alors sur la situation économique de ces unités, nécessairement dégradée compte tenu de l'impact des dépenses énergétiques dans la structure des coûts d'exploitation. Dans un tel contexte, les indicateurs de performance des unités côtières sont moins affectés que ceux des unités hauturières, les premières bénéficiant alors d'un effet d'aubaine par la mise en place des mesures $\mathrm{d}^{\prime}$ aides directes et indirectes.

De manière plus globale, en termes d'incidence sur les dépenses publiques, la pêche côtière a bénéficié d'un encadrement moins soutenu dans le financement du renouvellement de la flotte (GUYADER, 2007), en particulier dans les années 1980 (même si ce soutien n'a jamais été a priori justifié sur le plan économique). La part des subventions dans le financement de l'achat des navires de pêche en Bretagne atteignait $25 \%$ du montant de l'investissement sur la période 1981-1990 pour les unités de 16 à 25 mètres, exploitant principalement les pêcheries hors bande côtière. Cette part s'élevait à 18 \% pour les bateaux de 10-16 mètres, dont une partie se situe en bande côtière, et ne dépassait pas $4 \%$ chez les unités de moins de 10 mètres, totalement inféodées à la bande côtière.

Ces deux points peuvent tenir d'arguments afin de considérer la pêche côtière comme un point d'appui (non exclusif) pour une politique régionale de gestion durable du secteur halieutique.

D'autant plus que les mesures régionales de gestion des pêches sont désormais rendues possibles par l'évolution du régime juridique de la bande côtière. Ce régime $\mathrm{a}$, en effet, singulièrement évolué dans le cadre de la Politique Commune des Pêches, depuis $2002^{8}$, la délégation de pouvoir accordée par la Commission Européenne aux États facilitant la gestion décentralisée des zones côtières. Si les navires côtiers ne peuvent concurrencer les navires hauturiers en dehors de la bande côtière, la réciproque n'est pas vraie ; et ici, c'est moins la performance économique 
intrinsèque qui compte que la capacité de capture, d'où le risque de dommages irréversibles si des mesures préventives ne sont pas prises. Par conséquent, une politique régionale de gestion durable du secteur halieutique devrait prendre appui sur la flottille côtière, compte tenu, d'une part, de ses performances économiques (renforcées par une moindre dépendance à l'égard du facteur énergétique), et, d'autre part, de son plus faible rayon d'action la rendant plus dépendante de son territoire historique comparativement à la flotte hauturière.

\section{Bibliographie}

ADAM P., 1968, "Aspects économiques de la "surpêche" ", Revue Économique, $\mathrm{n}^{\circ}$ 1, pp. 130-171.

Berthou P., TAlidec C., Jezequel M., LesPagnol P., 1999, La flotte de pêche commerciale bretonne. Description des métiers et des flottilles. Etude cofinancée par la région Bretagne et l'IFREMER dans le cadre du XI ${ }^{e}$ Contrat de Plan État-Région. IFREMER Brest/Lorient, 127 p. + annexes.

Berthou P., Daurès F., GuYader O., Le Blond E., TAlidec C., ThéBaud O., 2006, Les flottilles de pêche côtière en Bretagne : caractéristiques et évolutions depuis 1990. Atelier International sur la « Régulation de l'accès aux ressources marines vivantes dans la zone côtière : expériences internationales et perspectives pour la Bretagne ", Plouzané, 20 au 20 janvier, IUEM.

Bihel J., LE FlOC'H P., 2006, Résultats des flottilles artisanales 2004/2005 - Note de synthèse. Observatoire Economique Régional des Pêches de Bretagne, 62 p.

BONCOEUR J., MESNIL B, 1999, "Surexploitation des stocks et conflits dans le secteur des pêches. Une discussion du "triangle des paradigmes" $\mathrm{d}^{\prime}$ Anthony CHARLES dans le contexte européen ", Informations et Commentaires, n 107, avril-juin, pp. 10-17.

Boncoeur J., Le Floc'H P., Giguelay T., LE GAllic B., 2000a, Les aides publiques à la flotte de pêche de la région Bretagne et leurs effets économiques, Cedem/IUEM/UBO, $111 \mathrm{p}$.

Boncoeur J., Coglan L., Le Gallic B., Pascoe S., 2000b, « On the (ir) relevance of rates measures of economic performance to small boats ", Fisheries Research, $\mathrm{n}^{\circ}$ 49, pp. 105-115.

Brook, A.-M., Price R., Sutherland D., Westerhund N., André C., 2004, Oil prices developments : drivers, economic consequences and policy responses, OECD, Economics Department Working papers, $\mathrm{n}^{\circ} 412,51 \mathrm{p}$.

Charles A., 1992, "Fishery conflicts. A unified framework », Marine Policy, n 4916, pp. 379-393.

CuRTIL O., 2005, in TALIDEC C. et al, 2005, Scénarios d'aménagement des activités de pêche dans la bande côtière bretonne, rapport d'activité, IFREMER, 207 p.

GUYADER O., 2007, Dynamiques d'exploitation et conditions d'évolution de la rente dans les pêches maritimes françaises, Thèse d'Habilitation à Diriger des Recherches, Université de Bretagne Occidentale, 104 pages.

IFREMER, 2006, Synthèse des flottilles de pêche 2004 - Flotte de Mer du Nord-Manche - Atlantique, Système d'Informations Halieutiques, 54 p.

INSEE, 2007, Tableaux de l'économie bretonne 2008, 185 p. 
LATOuChe K., LE Floc'H P., 2000, « Le comportement de mobilité de la flottille de pêche à une échelle régionale. Le cas de la flottille bretonne ", Revue d'Economie Régionale et Urbaine, $\mathrm{n}^{\circ} 1$, pp. 139-156.

LE FlOC'H P., MARTIN A., BOnCOEUR J., 2006. Les revenus à la pêche en Bretagne: situation en 2005 et évolution depuis 1998, Rencontres Halieutiques, 19-20 octobre 2006, Agrocampus, Rennes

Maurice J., 2001, Prix du Pétrole, Conseil d'Analyse Économique, Paris, 195 pages. OFIMER, 2006, Bilan annuel de production 2006 des pêches et de l'aquaculture, $101 \mathrm{p}$. SAlZ P., BuISMAN E., SMIT J., DE VOS B., 2006, Employment in the fisheries sector : current situation. Fish/2004/4, LEI BV, Framian BV. Final report, 185 p.

Union Européenne, 2000, Règlement (CE) n 1543/2000 du 29 juin 2000 instituant un cadre communautaire pour la collecte et la gestion des données nécessaires à la conduite de la politique commune de la pêche, Journal Officiel de l'Union Européenne, $16 \mathrm{p}$.

Union Européenne, 2001, Règlement (CE) $n^{\circ}$ 1639/2001 de la Commission du 25 juillet 2001 établissant les programmes communautaires minimal et étendu pour la collecte des données dans le secteur de la pêche et portant modalités d'application du règlement (CE) $n^{o}$ 1543/2000 du Conseil, Journal Officiel de l’Union Européenne, $63 \mathrm{p}$.

Union Européenne, 2006, Aide d'État $n^{\circ}$ C9 : 2006 (ex NN 85/2005), Fonds pour la prévention des risques liés aux activités du secteur de la pêche, Journal Officiel de l'Union Européenne, 5 p.

\section{Notes}

1 - La bande côtière est définie par l'espace maritime situé à moins de 12 milles nautiques de la côte (zone hachurée sur la figure 1). La révision de la Politique Commune des Pêches (PCP) en 2002 par la Commission Européenne a conduit à « un renforcement de la position de l'échelon régional et notamment de celle des Comités régionaux des pêches maritimes et des élevages marins » (CURTIL, 2005, p1).

2 - À l'échelle bretonne, les données économiques à la pêche sont collectées par voie d'enquête auprès des pêcheurs et par voie comptable auprès des centres de gestion à la pêche artisanale. L'IFREMER, responsable du système d'information halieutique (SIH), gère l'ensemble des travaux d'enquête portant sur l'activité des navires, leurs productions par espèce et les données économiques. La couverture statistique ne se limite pas à la Bretagne mais est étendue aux trois façades maritimes françaises (Manche-Mer du Nord, Atlantique et Méditerranée). La donnée de type comptable est transmise par les centres de gestion à l'Observatoire économique régional des pêches de Bretagne, dirigé par une organisation professionnelle à laquelle est associée le Cedem (Centre de Droit et d'économie de la mer) ainsi que le Comité Régional des pêches de Bretagne.

3 - La valeur ajoutée est égale à la différence entre la valeur de la production (assimilable au chiffre d'affaires en l'absence de variation de stocks) et celle des consommations intermédiaires (biens non durables et services extérieurs consommés dans le processus productif). Elle représente, en première approximation, l'excédent de la valeur créée sur la valeur détruite dans le cadre du processus productif. Cependant, elle ne tient pas compte de la perte de valeur subie par le capital fixe au cours de ce processus, du fait de l'usure ou de l'obsolescence (perte de valeur que la pratique comptable appréhende à travers la notion d'amortissement). C'est pourquoi il s'agit d'une valeur ajoutée brute. Les données comptables ne renseignent pas ici sur la valeur du capital. 
4 - La valeur ajoutée brute totale de la Région Bretagne est évaluée à 73511 millions d'euros pour l'année 2005, soit 4,4\% de la VAB de la France métropolitaine.

5 - Le montant de l'indemnisation est intégré aux frais communs dans le compte d'exploitation des entreprises de pêche artisanale (Observatoire Economique Régional des Pêches, 2006).

6 - L'Etat français a participé au financement du FPAP. Cette intervention financière a fait l'objet, le 19 avril 2006, de l'ouverture de la procédure formelle d'examen de la part de la Commission Européenne (Union Européenne, 2006). La Commission Européenne considère, en effet, que la participation de l'Etat français à hauteur de 65 millions d'euros au FPAP relève d'une mesure discriminatoire à l'égard des autres secteurs d'activité économique à l'échelle européenne. Une enveloppe supplémentaire de 22 millions d'euros a été versée par l'Etat au FPAP au second semestre 2006. La contribution financière des pêcheurs adhérents s'élève à 4 millions d'euros.

7 - La littérature économique sur les pêcheries emploie fréquemment le terme de communauté (dans le sens d'une collectivité) pour définir l'industrie des pêches maritimes localisée à une échelle régionale. Ainsi, P. ADAM précise dans son article de 1968 que ... " beaucoup d'industries des pêches sont le fait de communautés qui n'ont pas d'autres possibilités pour gagner leur vie et qui participent mais ne profitent que de façon très indirecte aux mouvements économiques globaux (p. 136) ». Il est, en effet, rare de constater un impact économique fort de cette industrie à un niveau géographique local même sur des territoires concentrant une partie élevée d'une flotte de pêche (cas de la Bretagne). La justification du maintien des emplois à la pêche tient davantage à des considérations d'ordre social ou de paix sociale (BonCOEUR et MESNIL, 1999) qu'à des arguments d'efficacité économique présentant l'industrie du poisson comme une activité essentielle à l'économie régionale (hormis le cas de régions très dépendantes de la pêche situées au Pérou, en Chine, en Norvège ou en Islande).

8 - L'article 9 du Règlement (CE) n ${ }^{\circ}$ 2371/02 (du Conseil du 20 décembre 2002, JOCE $\mathrm{n}^{\circ} \mathrm{L}$ 358 du 31 décembre 1992, p. 59), précise que « Un État membre peut adopter des mesures non discriminatoires pour la conservation et la gestion des ressources de pêche et pour minimiser les incidences de la pêche sur la conservation des écosystèmes marins dans la zone des 12 milles marins à partir de ses lignes de base, pour autant qu'aucune mesure de conservation et de gestion n'ait été adoptée par la Communauté spécifiquement pour cette zone (...)» (CURTIL, 2005, p. 332). 